Meta

Journal des traducteurs

Translators' Journal

\title{
Je ne file pas très bien aujourd'hui ou Comment ne pas traduire le verbe " to feel "
}

\section{Geneviève Quillard}

Volume 30, numéro 2, juin 1985

URI : https://id.erudit.org/iderudit/003898ar

DOI : https://doi.org/10.7202/003898ar

Aller au sommaire du numéro

Éditeur(s)

Les Presses de l'Université de Montréal

ISSN

0026-0452 (imprimé)

1492-1421 (numérique)

Découvrir la revue

Citer cet article

Quillard, G. (1985). Je ne file pas très bien aujourd'hui ou Comment ne pas traduire le verbe « to feel ». Meta, 30(2), 168-173.

https://doi.org/10.7202/003898ar d'utilisation que vous pouvez consulter en ligne.

https://apropos.erudit.org/fr/usagers/politique-dutilisation/ 


\section{JE NE FILE PAS TRĖS BIEN AUJOURD'HUI \\ OU \\ COMMENT NE PAS TRADUIRE LE VERBE «TO FEEL "}

Au cours de leur apprentissage d'une langue seconde, il arrive fréquemment que les élèves produisent des énoncés calqués sur la langue de départ, c'est-à-dire qu'ils agissent comme si les deux codes linguistiques correspondaient parfaitement. Leur faire comprendre que chaque langue est régie par des lois qui lui sont propres et que l'on ne peut impunément "plaquer " un système linguistique sur un autre est une entreprise de très longue haleine, source de bien des sentiments de frustration, tant chez les élèves que chez les enseignants.

Ainsi il est souvent difficile d'amener les étudiants, même ceux d'un niveau avancé, à concevoir que le découpage du réel puisse s'effectuer d'une autre façon dans une autre langue et que, par conséquent, un terme puisse posséder une extension différente en anglais et en français, par exemple ${ }^{1}$. 
La tâche est encore plus malaisée si c'est le terme de la langue d'arrivée qui présente la plus petite extension. Ainsi la traduction en français du verbe " to feel " pose aux anglophones des difficultés considérables. Combien de fois les professeurs de français langue seconde n'ont-ils pas lu ou entendu des énoncés fautifs tels que : "Elle se sent bien qu'elle n'aurait pas dû dire cela ", "Je sens très fatigué aujourd'hui ", "Je ressens que vous avez raison ", etc. ? Il faut reconnaître que la traduction de ce "petit » verbe si fréquemment utilisé est moins évidente qu'elle ne le paraît de prime abord, et ce pour diverses raisons :

1. Il peut exprimer soit une action, soit un état ${ }^{2}$ et peut donc posséder deux types de sujets : un sujet animé ("The doctor is feeling her pulse ") ou un sujet inanimé ("He says it feels normal ") ${ }^{3}$. Or le français sépare nettement ces deux catégories ${ }^{4}$, contraignant le locuteur à utiliser des termes différents selon que le statut est un être humain ou un objet (dans l'usage «normal » bien entendu). primer :

2. Il possède une structure sémantique relativement complexe puisqu'il peut ex-

a) le toucher, comme dans les exemples donnés ci-dessus.

b) une sensation : "She felt a dull ache in her throat", "He felt a warm spurt of blood on his face ";

c) un sentiment : "We felt ashamed ", "I feel good about my work", "He felt that they were conducting a vendetta against him ", "I feel that soon I won't be able to stand the heat any more".

Le français, lui, distingue tous ces domaines les uns des autres et exige donc un terme différent selon qu'il s'agit du toucher, AU SENS PROPRE DU TERME, d'une sensation ou d'un sentiment ${ }^{5}$.

3. Les structures syntaxiques compatibles avec ce verbe sont multiples en anglais. Or si la sélection du mot juste en français relève du domaine sémantique, comme nous venons de le voir ( 1 et 2), elle relève également, et plus encore semble-t-il, de la syntaxe. Il existe donc en français une double contrainte, sémantique et syntaxique, tandis qu'en anglais il n'y en a aucune.

4. Ce verbe apparaît assez fréquemment sous des formes telles que : « to feel like» ou " to feel as if ", qui donnent lieu à des traductions tout à fait différentes.

Loin de nous la prétention de résoudre tous les problèmes dans le cadre de ce premier article et de fournir une liste exhaustive des traductions de toutes les occurrences possibles du verbe " to feel ". Notre but, avouons-le tout de suite, est extrêmement modeste et surtout très pragmatique : d'une part, à défaut de trouver les réponses, nous aimerions poser les questions, c'est-à-dire tracer les grandes lignes des difficultés que pose ici l'opération de transcodage ; d'autre part, et c'est là en définitive que réside l'essentiel, nous voudrions fournir aux élèves ou aux étudiants anglophones, en nous en tenant aux occurrences les plus fréquentes ${ }^{6}$, un outil qui sera suffisamment efficace pour leur permettre de sélectionner assez rapidement et avec un risque d'erreur minimal le mot qui s'adapte au contexte dans lequel il s'inscrit. Nous serons donc amenée à opérer un certain nombre de simplifications : c'est inévitable. Mais si, dans un premier temps, elles permettent d'éliminer des fautes qui ne sont que trop courantes, ou d'en réduire le nombre, cet article aura atteint le but que nous lui assignons.

Une autre précision s'impose : nous nous intéresserons surtout à l'aspect « actif » du verbe, dans le cadre de cet article, car c'est à ce niveau que se situent la plupart des erreurs commises par les étudiants.

Étant donné ce qui a été dit plus haut (voir 1), il faut commencer par établir deux catégories principales, selon que le verbe anglais exprime un état ou une action. 
A : TO FEEL + SUJET INANIMÉ (ÉTAT)

Les traductions les plus fréquentes semblent être les suivantes ${ }^{7}$ :

a) ÊTRE : "The doctor says her pulse feels normal »

"Le docteur dit que son pouls est normal "

"It feels strange to be back here"

"C'est étrange d'être de retour ici »

b) DONNER L'IMPRESSION/AVOIR L'AIR :

"The basement feels damp"

«Le sous-sol donne l'impression d'être humide/a l'air d'être humide »

"The house felt empty"

"La maison donnait l'impression d'être vide/avait l'air d'être vide "

c) ON DIRAIT (AURAIT DIT) QUE/AVOIR L'IMPRESSION

"It feels like rain"

«On dirait qu'il va pleuvoir " ou "J'ai l'impression qu'il va pleuvoir»

Il faut noter qu'avec l'utilisation de ces deux expressions s'opère un changement de point de vue, puisqu'elles ne peuvent être associées qu'à des agents animés.

B : TO FEEL + SUJET ANIMÉ (ACTION)

a) Il faut établir une catégorie à part pour tout ce qui a trait au toucher, au sens propre du terme répétons-le, puisque dans ce cas il semblerait que " to feel " se traduira toujours par "toucher" ou "palper" ou l'un de leurs synonymes.

b) Il est également possible de mettre à part les occurrences " to feel good/bad $A B O U T$ SOMETHING " ou autres expressions sémantiquement très proches de cellesci. Il semble en effet qu'elles peuvent toujours se rendre par :

ETTRE CONTENT DE/RAVI DE, etc.

NE PAS ÊTRE CONTENT DE/REGRETTER

"I feel good/bad about my work»

"Je suis content/mécontent de mon travail "

"I feel bad about not phoning you"

"Je regrette de ne pas vous avoir téléphoné "

c) Pour le reste, les termes qui surviennent le plus fréquemment en français sont : sentir, se sentir, avoir l'impression, ressentir et éprouver ${ }^{8}$. L'utilisation de ces termes est régie, nous l'avons vu ci-dessus, par des contraintes syntaxiques plus encore que par des contraintes sémantiques. Il paraît donc judicieux de commencer par analyser la structure syntaxique de la phrase anglaise (d'autant que la syntaxe est toujours plus «palpable " que la sémantique), ce qui permettra d'opérer un premier tri, voire, dans un assez grand nombre de cas, de sélectionner très rapidement le terme qui convient en français, sans même avoir besoin de recourir à une analyse sémantique. Ce n'est que lorsque cette grille laissera à l'élève plusieurs possibilités qu'il devra effectuer une seconde démarche et se livrer à une analyse sémantique. 
Analyse syntaxique

Elle peut s'effectuer au moyen de la grille suivante :

\begin{tabular}{lcccc}
\hline & $\begin{array}{c}\text { feel }+ \text { adv. } \\
\text { loc. adv. }\end{array}$ & feel + adj. & $\begin{array}{c}\text { feel }+ \text { COD. } \\
\text { [gr. nom. }\end{array}$ & feel + prop. \\
\hline SE SENTIR & + & + & & \\
\hline RESSENTIR & + & & + & + \\
ÉPROUVER & & & + & + \\
\hline SENTIR & & & + \\
\hline AVOIR & & & & + \\
L'IMPRESSION & & & & + \\
\hline
\end{tabular}

Cette première grille permet, on le voit de procéder à un certain nombre d'éliminations du premier coup d'œil. À partir du moment, par exemple, où le verbe anglais est suivi d'un adjectif ou d'un adverbe, il n'y a plus de doute possible : en français le locuteur sera contraint d'utiliser "se sentir "10. En fait, on s'aperçoit que l'analyse sémantique ne s'impose que dans un cas seulement, celui du choix entre sentir et ressentir/ éprouver, lorsque le verbe est accompagné d'un groupe nominal.

\section{Analyse sémantique}

Si l'on distingue le physique du mental (que l'on pourrait également qualifier d'affectif), on obtient la grille suivante :

\begin{tabular}{lcc}
\hline & mental affectif & physique \\
\hline RESSENTIR & + & + \\
EPPROUVER & + & + \\
\hline
\end{tabular}

Ici encore, malheureusement, il s'agit d'une description assez grossière, qui aurait besoin d'être affinée à l'aide de minutieuses analyses sémiques, car, si RESSENTIR et ÉPROUVER peuvent effectivement exprimer des sensations (ressentir la soif ou la faim, ressentir ou éprouver des douleurs), il n'est pas toujours possible de les utiliser lorsque l'on parle de quelque chose de physique. Ainsi on ne peut ressentir ou éprouver un coup sur la tête ou la bosse que l'on vient de se faire sur le front en rencontrant sur son chemin un poteau télégraphique (bien que l'on ait le droit de ressentir ou d'éprouver les douleurs qui s'ensuivent !), et l'on ne peut non plus ressentir ou éprouver une main sur son genou dans une salle de cinéma obscure. Un tremblement de terre, cela se sent uniquement. Un choc, en revanche, peut être SENTI ou RESSENTI/ÉPROUVÉ, mais le choix du verbe n'est alors pas indifférent, car dans le premier cas il s'agit d'un ébranlement externe, tandis qu'il est purement interne dans le second.

En attendant des descriptions et des analyses plus poussées, qui permettraient de cerner avec précision les différentes utilisations des verbes ressentir et éprouver dans le domaine physique, le plus sage paraît être de conseiller aux étudiants de réserver ces deux verbes au domaine du mental (affectif). Sauf erreur de notre part, si l'on s'en tient à SENTIR pour tout ce qui est physique et à RESSENTIR/ÉPROUVER pour le reste, on ne court pas le risque de produire des énoncés inacceptables en français. 


\section{C : TO FEEL LIKE ET TO FEEL AS IF}

Avant de terminer ce bref tour d'horizon il n'est peut-être pas inutile de voir comment peuvent se traduire les deux expressions dont les occurrences sont assez nombreuses : TO FEEL LIKE et TO FEEL AS IF.

1 : TO FEEL LIKE

a) accompagné d'un sujet animé : avoir envie de — cela me (te/lui, etc.) dit — cela me (te/lui, etc.) tente

"They feel like going to the movies"

"Ils ont envie d'aller au cinéma » ou

"Ça leur dit (ça les tente) d'aller au cinéma "

"I feel like a beer"

«J'ai envie d'une bière »11

b) accompagné d'un sujet inanimé : donner l'impression - faire

"What does it feel like to be married?"

"Qu'est-ce que cela te (vous) fait d'être marié ?"

"It felt like being back home»

"Cela me donnait l'impression d'être de retour chez moi "

2 : TO FEEL AS IF

a) accompagné d'un sujet animé : avoir l'impression

"He felt as if he were falling"

"Il avait l'impression de tomber"

"She felt as if the whole world were against her"

"Elle avait l'impression que le monde entier était contre elle»

b) accompagné d'un sujet inanimé : on dirait que - avoir l'impression

"It feels as if she wants me"

"J'ai l'impression qu'elle me désire»

"It feels as if it is trying to rain"

"On dirait qu'il va (veut) pleuvoir"

"It feels like spring"

"On dirait que c'est le printemps" ou "On a l'impression d'être au printemps". (En réalité, dans ce cas, l'expression qui viendrait tout de suite à l'esprit d'un francophone serait probablement : "Ça sent le printemps!")

Il faut noter une fois de plus ici le changement de point de vue qui s'établit en français dans la mesure où ces deux expressions expriment le point de vue de quelqu'un.

Une bonne partie du travail reste à faire, notamment dans le domaine sémantique. Pourtant cette ébauche n'aura pas été totalement inutile si elle parvient à donner aux étudiants certaines des réponses qui leur manquent actuellement, En outre, il n'est pas interdit d'espérer que cette modeste étude incitera quelqu'un d'autre à revenir sur ce sentier tortueux, taillé à grands coups de machette, pour le transformer en une voie royale au tracé rectiligne. Comme nous filerions bien dans ce cas! 
Notes

1. Voir J.P. Vinay et J. Darbelnet (1958) : Stylistique comparée du français et de l'anglais, Montréal, Beauchemin, pp. 63-69.

2. "But for SMELL, FEEL and TASTE, there is no special corresponding activity verb, so these verbs have to do duty for the state meaning and the activity meaning. "Geoffrey Leech, Jan Svartvik (1979) : $A$ Communication Grammar of English, London, Longman, p. 70.

3. Nous avons emprunté ces exemples à G. Leech et J. Svartvik, id., p. 73.

4. Bien qu'en fait ce soient les deux aspects d'une même chose : l'un interne (I feel hot), l'autre externe (It feels hot).

5. Ce qui explique pourquoi nous avons classé séparément toucher et sensations physiques, bien que celles-ci relèvent du même sens.

6. Nous voulons insister sur ce point et préciser que, lorsque nous parlons d'occurrences fréquentes, nous nous référons, non à des statistiques probablement encore inexistantes, mais aux productions de nos étudiants.

7. Il serait intéressant de déterminer avec précision les conditions d'utilisation de ces diverses traductions (sont-elles toujours interchangeables ou non?), mais pour ce faire il faudrait effectuer des analyses sémiques assez approfondies, qui n'entrent pas dans le cadre que nous nous sommes fixé pour cet article.

8. Pour simplifier notre tâche ainsi que celle des élèves ou des étudiants, nous considérerons ici comme parfaitement synonymes les verbes SENTIR et AVOIR L'IMPRESSION d'une part, RESSENTIR et ÉPROUVER d'autre part.

9. SE SENTIR peut être suivi d'un indicatif, certes (Il se sentait mourir ; Il se sent renaître). De même des énoncés du type : " Il se sentait le cœur léger " ou "Il se sentait un autre homme " ne violent aucunement le code linguistique français. Si nous avons décidé de ne pas en tenir compte, c'est tout simplement parce qu'ils ne nous est jamais arrivé de les lire ou de les entendre chez nos étudiants.

10. Nous ne prenons pas en considération les cas particuliers, nous l'avons déjà dit. Il y a, nous en sommes consciente, des occurrences de TO FEEL accompagné d'un adjectif ou d'un adverbe qui ne se rendront nullement en français par SE SENTIR. C'est le cas par exemple de : "I felt faint " qui se traduirait par "J'ai eu un malaise" ou "Je me suis trouvé mal ".

11. On remarque ici un changement de point de vue. 